\title{
Auditory training methods in children with psychiatric diagnosis: an integrative literature review
} Humberto de Oliveira Simões ${ }^{1}$
https://orcid.org/0000-0003-3544-8262

Sthella Zanchetta ${ }^{2}$ https://orcid.org/0000-0001-9171-6116

Erikson Felipe Furtado ${ }^{3}$ https://orcid.org/0000-0001-8006-7077

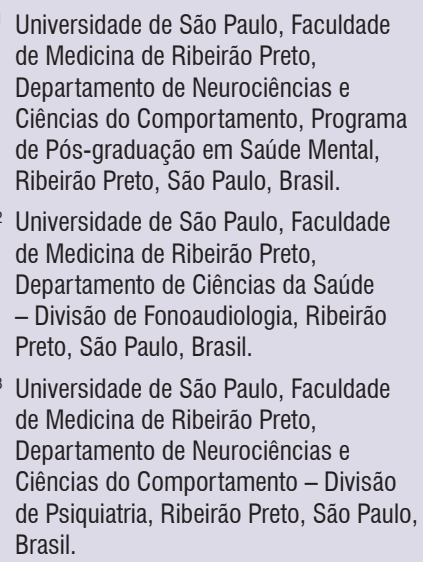

Research support source: Higher Education Personnel Improvement Coordination (CAPES, from the acronym in Portuguese)

Conflict of interests: Nonexistent

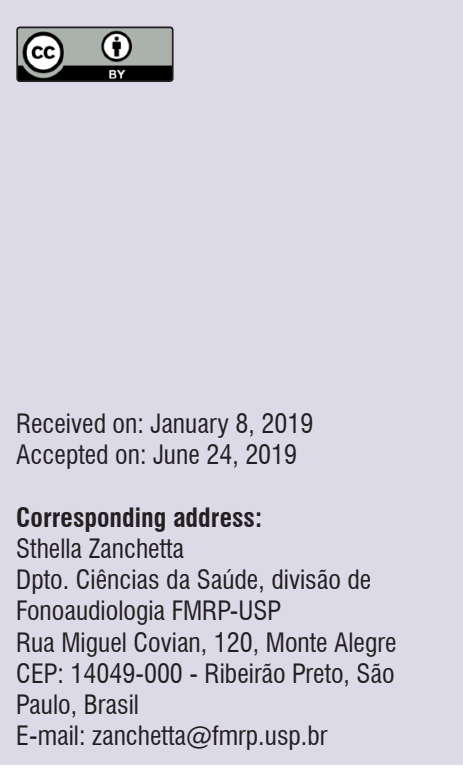

\section{ABSTRACT}

Purpose: to identify auditory training approaches used in the pediatric population with psychiatric diagnoses and to characterize the contexts in which the intervention took place, as well as protocols and discernment of the results.

Methods: a search was carried out in four databases, using the fixed terms "auditory training" OR "auditory rehabilitation". Regarding the sampling, there was a considerable range of diagnoses, signs and symptoms, including the most common ones. Articles published until August 2018 whose population did not present hearing loss were selected; articles whose subjects had made use of any personal sound amplification products, presented otitis or had any isolated diagnosis of auditory processing disorder, were excluded.

Results: 16 articles out of the 103 references found, met the inclusion criteria. The samplings studied were children and adolescents with learning, language or reading disorders, dyslexia, attention deficit hyperactivity disorder, autism spectrum disorder and schizophrenia. The bottom-up intervention and the combined approach (bottom-up and top-down), in the formal context, were the most frequent approaches, whose results led to the improvement in linguistic, metalinguistic and auditory skills. The amount and frequency of sessions, as well as their duration varied.

Conclusion: the heterogeneity of auditory training techniques diversified the results. However, it seems there is a potential for recommending auditory training in the reviewed population.

Keywords: Child Psychiatry; Psychiatric Diagnoses; Signs and Symptoms; Auditory Training; Auditory Processing 


\section{INTRODUCTION}

Auditory processing, in its classical definition, refers to what we do with what we hear'. It's a necessary function for the development of social interaction skills, learning aspects, speech and language ${ }^{2}$.

In the perspective of changes in the auditory behavior in the auditory processing, the training of auditory skills necessary to process acoustic information (which, when altered, may be characterized as Central Auditory Processing Disorder) is defined as a set of strategies aimed at activating the auditory system and its associations, positively changing the auditory behavior and the central auditory nervous system (CANS) ${ }^{3-5}$. It must be considered that the auditory processing not only includes the auditory system's mechanism, both peripheral and central, but also is influenced by top-down mechanisms, such as language, attention, memory and executive functions ${ }^{6}$.

The main components of the therapeutic intervention approach in auditory training (AT) propose a development by means of bottom-up and top-down mechanisms ${ }^{7}$. Bottom-up deals with neurophysiological coding of auditory stimuli, centripetally directed, form the auditory nerve to the cortical areas, with approaches that make the acoustic signal clearer and/ or improve the acoustic environment, including assistive listening systems, clear discourse and improved room acoustics $^{6-8}$. According to the authors, in top-down the supramodal processes to hearing (e.g., memory, attention and language) act directly upon the analyses of the acoustic codes, working with language, cognitive and metacognitive strategies, as well as educational interventions with strategies for learning and/or facilitating in the work/home environment, offering compensating methods to minimize deficit in functional hearing.

Two contexts may be identified for the carrying out of AT, i.e., the means to be used ${ }^{8,9}$. The first is denominated formal AT, which is performed in an acoustically controlled manner with the help of electroacoustic equipment or computer programs. The second is the informal AT, defined as an intervention program executed in a therapy room or under the attention of a teacher or someone responsible for the person, without the need of sophisticated equipment.

Regardless of the mechanism in use for AT and the context in which it was carried out, when it's finished it's all-important to determine the effectiveness of the program that has been used ${ }^{5}$, either by means of behavioral assessment or electrophysiologic auditory tests. Effectiveness refers to the ability of producing and maintaining an effect, i.e., being both efficient and effective. In this sense, the studies with cortical evoked potentials, before and after AT, have shown changes in morphology, latency and amplitude, which were considered to be due to a new reorganization of the central auditory nervous system, being thus interpreted as evidence of plasticity in the neural system ${ }^{4,5,9-13}$.

Regarding the psychiatric diagnoses in children and adolescents, their current estimates are in the order of $13.4 \%$, based on community studies from 27 countries of all continents ${ }^{14}$. According to data from the U.S. Census Bureau, which in 2014 assessed approximately 1.8 billion people worldwide, ranging from 5 to 19 years old, 241 million of them were reckoned to present some sort of mental disorder. In Brazil, literature does not point to an epidemiologic estimate that would represent the whole country, but it does indicate that 7 to $14 \%$ of Brazilian children and teenagers present some psychiatric disorder ${ }^{15}$.

Most of child and adolescent psychiatric diagnoses involve a combination of signs and symptoms in four domains: emotional symptoms, behavioral problems, development delay, and relationship difficulties (to which schizophrenia and anorexia nervosa are exceptions ${ }^{16}$ ).

The present amount of diagnoses of child and adolescent mental disorders brought about an increase in requests for treatment resources, such as the use of drugs and/or alternative medicine (as the psychotherapeutic), educational services and special services ${ }^{17,18}$.

Studies demonstrate that in face of the child and adolescent psychiatric issue, many of the samples in which the subjects present spoken language disorders, written language disorders, Autism Spectrum Disorder (ASD), Attention Deficit Hyperactivity Disorder (ADHD), and schizophrenia, are related to some change in the auditory information processing ${ }^{10,13,19-24}$.

Aiming at identifying the AT methods used in the population of children with psychiatric diagnoses, this review also intends to characterize the types of intervention, their protocols (including, but not limited to, the instrument adopted to control effectiveness), as well as the discernment of the results.

\section{METHODS}

\section{Search strategy}

The integrative literature review was carried out through the search in four databases, which are vastly comprehensive in terms of national and international 
periodical indexes, namely: PubMed, SciELO, LILACS and PsycINFO. All the references to each and everyone of the articles were checked for inclusion according to the defined criteria.

After some attempts and reviews of the results obtained in each database, considering the losses resulting from the use of the MeSH and DeCS full terms, it was decided to use more encompassing expressions for the terms that referred to the kind of participants and the type of intervention, which were then used in all search interfaces.

The searches in the abovementioned databases were performed by means of the fixed terms "auditory training" OR "auditory rehabilitation", in order to find the greatest number of articles with the intended subject. The other keywords related to psychiatric diagnoses, signs and symptoms in the child population, used for cross-referencing, had as main basis meta-analysis published with the theme of prevalence of mental disorders in children and teenagers ${ }^{14}$, following the diagnostic standards of the International Classification of Diseases (IDC) and the Diagnostic and Statistical Manual of Mental Disorders (DSM). These are the words that were used: autism; ADHD; attention deficit hyperactivity disorders; attention deficit; hyperactivity disorder; language disorders; dyslexia; depression; anxiety disorders; schizophrenia; obsessive compulsive disorder; posttraumatic stress disorder; separation anxiety disorder; social anxiety disorder; selective mutism; conduct disorder; eating disorder; anorexia; bulimia; psychosis; alcohol; drug; encopresis; enuresis; disruptive disorder; Rett Syndrome; Asperger Syndrome; tic disorder; phobia; mental retardation. In all databases, the keywords were presented in English.

For the reading of summaries and full articles, the following inclusion criteria were adopted: a) studies carried out with humans whose population were of children and teenagers (under 18 years old), associated with at least one psychiatric diagnosis; b) auditory training as a rehabilitation process; c) publications without an initial date limit and up to August $15^{\text {th }}$, 2018; d) publications in either Portuguese, English or Spanish; e) studies of the following types: case series, longitudinal, transversal, case-control, cohort and clinical trial.

For the exclusion of articles, the following criteria were adopted: a) studies involving animals or adult and elderly population (above eighteen years old); b) children or teenagers without child psychiatric diagnosis, signs or symptoms; c) studies of the following types: narrative review, systematic review, meta-analysis, dissertation, thesis, letter, editorial, commentary, case report; d) studies carried out with child or adolescent subjects with hearing loss of any type or degree, using any sound amplification device (Personal Sound Amplification Product, cochlear implant, bone-anchored hearing aid and/or Frequency Modulation System), ear infection (otitis) and isolated Central Auditory Processing Disorder (CAPD) diagnosis.

\section{Data analysis}

the selected scientific articles were studied by two speech-language pathologists who had to fill in a spreadsheet with the following information: a) author and date; b) level of evidence and degree of recommendation; c) description of cases; d) description of method of auditory training (type, number of sessions, frequency, approach and context); e) measurement of effectiveness of the intervention; f) main outcomes.

In order to guide the interpretation, regarding the methodological design of each article, the classification criterion based on the levels of evidence, from 1 to 5 , according to what is proposed by the Oxford Centre for Evidence-based Medicine - Levels of Evidence, was adopted $^{25}$. Afterwards, to identify whether the findings could be generalized, each text was further classified as to its level of recommendation. It should be noted that, in this classification system, the "Therapy/Prevention, Aetiology/Harm" column was used. The spreadsheets of both speech-language pathologists were then compared. Whenever there was a difference between them in any of items, the article was fully read together. In case the difference remained, a third professional in the field was called in.

\section{LITERATURE REVIEW Main findings}

Based on the selected terms, 103 references were found; of these, 58 summaries were examined. During the reading, 17 were excluded $(30.3 \%)$, for the cases included hearing loss, use of sound amplification devices, presence of otitis and/or isolated Central Auditory Processing Disorder diagnosis; another 14 summaries $(25.0 \%)$ were excluded for not focusing on child psychiatric diagnoses or signs and symptoms. In the end, 18 articles (17.6\%) were selected for full reading. Two other articles were yet excluded, one for being a brief communication, and the other for not 
having in its methodologyinformation about the type of auditory training. The selection was thus concludedwith 16 articles.
The flowchart of the screening of references from the databases until the final selection of scientific articles is shown on Figure 1.
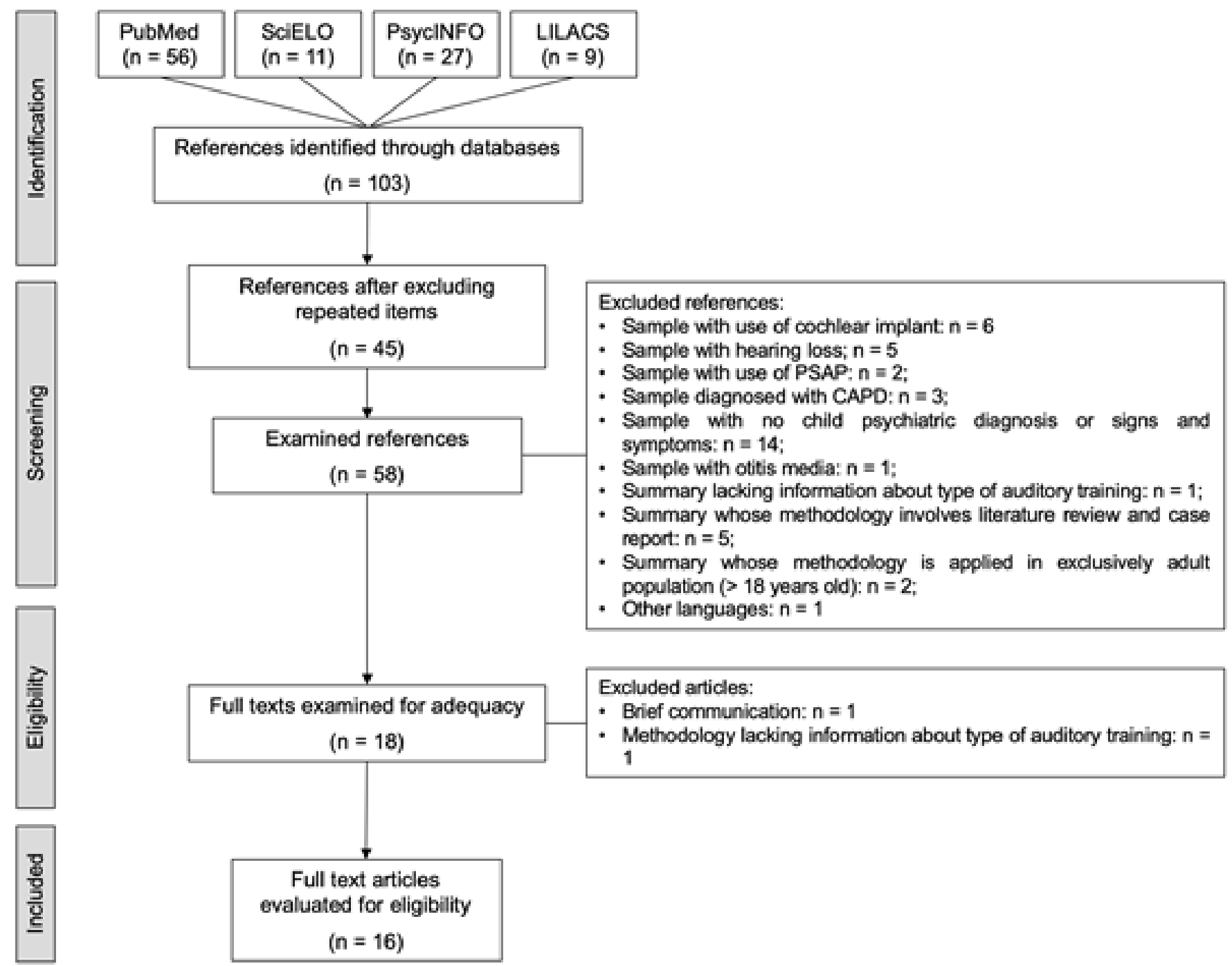

Figure 1. Flowchart of collection from the databases 
The year of publication of the articles ranges from 1968 to 2018. There has been noticed no major predominance of publications in any specific year.

As for the keywords that made up the data search for the combinations of child psychiatric diagnosis or signs and symptoms, there was a greater incidence of articles found under "dyslexia", "autism" and "learning disorders", respectively. The following keywords, on the other hand, returned no articles whatsoever: "obsessive compulsive disorder", "oppositional defiant disorder", "posttraumatic stress disorder", "separation anxiety disorder", "social anxiety disorder", "selective mutism", "eating disorders", "anorexia", "bulimia", "alcohol", "encopresis", "enuresis", "disruptive disorder", "Rett Syndrome", "tic disorder" and "phobia".

In the sampled articles, the various authors worked on four different group compositions, namely: treatment groups with a specific condition and control groups (with typical development or with some condition), either having or not received intervention, which were altered according to proposed outline. The variations in the outline of the samplings were as follows: group with a condition which received intervention vs. group with typical development without intervention ( $\mathrm{n}=7$; $43.75 \%)$; just the group with a condition that received intervention $(n+5 ; 31.25 \%)$; group with a condition that received intervention vs. control group with a condition without intervention vs. group with typical development without intervention ( $n=2 ; 12.5 \%)$; group with a condition that received intervention vs. control group with a specific condition that did not receive intervention ( $n=1 ; 6.25 \%$ ); group with a condition that received intervention vs. control group with psychiatric condition that received a placebo intervention $(n=1$; $6.25 \%)$.

The age of the subjects ranged from children 3 years old to groups that included teenagers and adults, above the age of 18 . The diagnoses of the cases used by the authors in the studied articles varied; however, there was a predominance of children with learning disorder $(n=4)$ and dyslexia $(n=4)$, in addition to reading disorder $(n=2)$, Autism Spectrum Disorder $(n=3)$, schizophrenia $(n=1)$, Specific Language Impairment $(n=1)$ and ADHD $(n=1)$.

The degree of recommendation and level of evidence in the articles, according to the adopted classification, varied between $B / 2 B(n=1 ; 6.25 \%)$, $B / 2 C(n=14 ; 87.5 \%)$ e $C / 4(n=1 ; 6.25 \%)$. The relation of diagnoses and signs and symptoms to the levels of evidence and degrees of recommendation of the selected articles may be seen on Figure 2 .

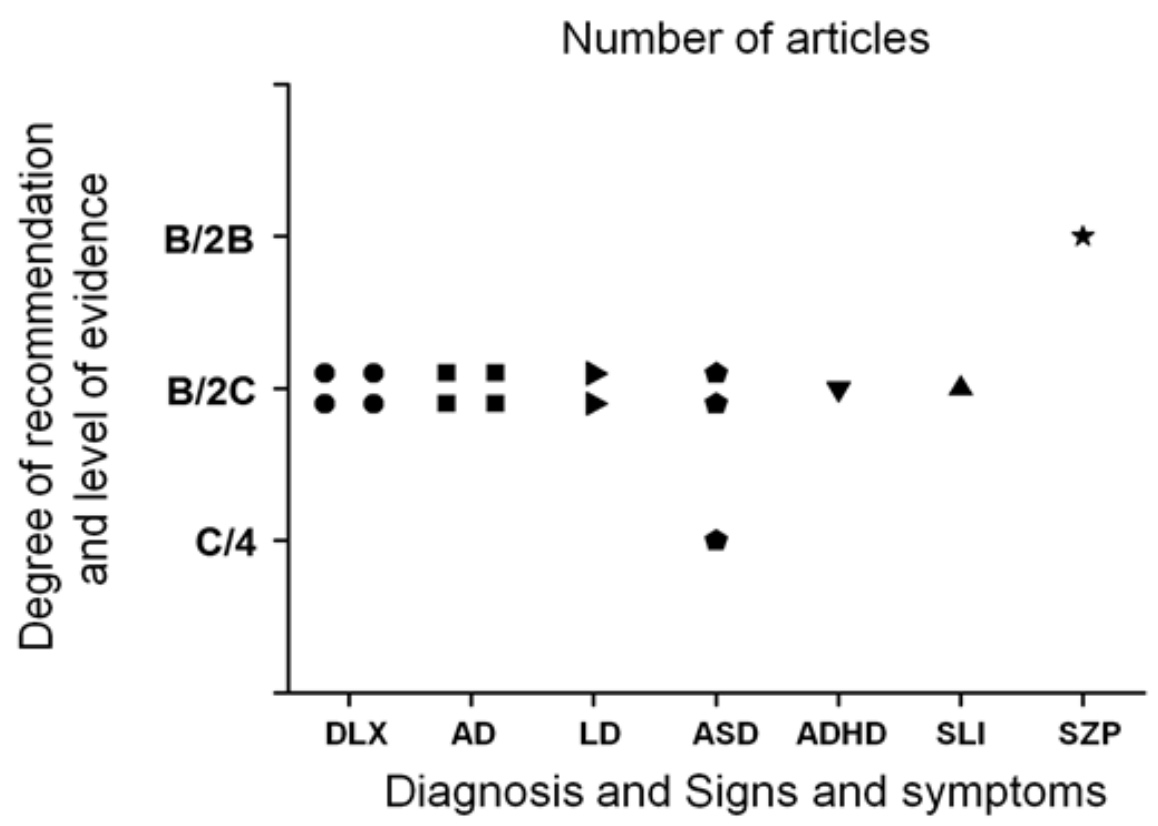

Legend: DLX= dyslexia; $A D=$ attention disorder; $L D=$ language disorder; $A S D=$ Autism Spectrum Disorder; $A D H D=$ attention deficit hyperactivity disorder; $\mathrm{SLI}=$ specific language impairment; $\mathrm{SZP}=$ schizophrenia.

Figure 2. Diagnoses and signs and symptoms in relation to the level of evidence and degree of recommendation of the selected articles $(n=16)$ 
The auditory training approaches, the number of sessions, the duration and frequency of sessions were heterogeneous, and in a number of articles some of these variables were not described. Seven articles used the bottom-up AT approach (37.5\%), four used the top-down approach (25.0\%), five used both bottom-up and top-down (31.25\%) and in only one it wasn't possible to identify it $(6.25 \%)$. The formal context of applying AT prevailed ( $n=14 ; 87.5 \%)$, while in one only the informal context was identified $(6.25 \%)$; in one article $(6.25 \%)$ it wasn't possible to specify the context, due to the lack of description in the methodology.

In all articles there was a control of the effectiveness of the adopted auditory training, by means of monitoring before and after intervention. The different means of control were made up of questionnaires, language skills assessments, and behavioral and electrophysiologic tests of auditory processing, which in most cases differed from the moments of evaluation.

When the characteristics of the child psychiatric diagnoses or signs and symptoms of the articles are associated with the adopted approach and context of auditory training, their alternation is noticed. In the conditions of learning disorder and dyslexia, both the bottom-up and the top-down approaches were used separately, as well as together, in the context of formal training; for the diagnosis of ASD, both bottom-up and top-down approaches were used, separately, in the context of formal training; when there was reading disorder, training occurred with simultaneous bottom-up and top-down approaches, in both formal and informal contexts; for ADHD diagnosis, the bottom-up training was used in a formal context, and so it was in the case of Specific Language Impairment; lastly, for the diagnosis of schizophrenia, the bottom-up approach was used in a formal context.

In the main results of the articles, the work carried out among students with learning and/or reading disorders in face of a proposed auditory training program affected both the perception of sound and its cortical representation. They have also shown to have plasticity in the neural codification of the speech sounds in the cortex, with better performance in phonologic awareness and phonemic abilities. The improvement in the performance in auditory behavior may be attributed to plasticity ${ }^{10,26-28}$. In the studies involving dyslexic subjects, the observed improvements were greater agility in accomplishing tasks, speed and precision in identifying words, improvement in reading tests performance, phonologic awareness and evolution in phonologic decoding, as well as in auditory behavior tests ${ }^{11,29,30}$.

In the samples of the three studies ${ }^{12,20,24}$ with subjects diagnosed with ASD, those children presented an improvement after intervention for auditory sensitivity, dichotic hearing, cortical processing and decrease in latency of the brainstem auditory evoked potential for speech sounds. For those diagnosed with $A D H D^{22}$, the only study found points to a decrease in impulsiveness in auditory detection tasks.

In the study which worked with the schizophrenia diagnosis ${ }^{23}$, the results achieved by the subjects in face of AT showed improved in global cognition, verbal memory and problem-solving, as well as in the functioning of the motivational system, proving to be more efficient in the fast processing of verbal stimuli.

The identification and description of the 15 articles, according to the questions used in the research, may be consulted in Table 1. 


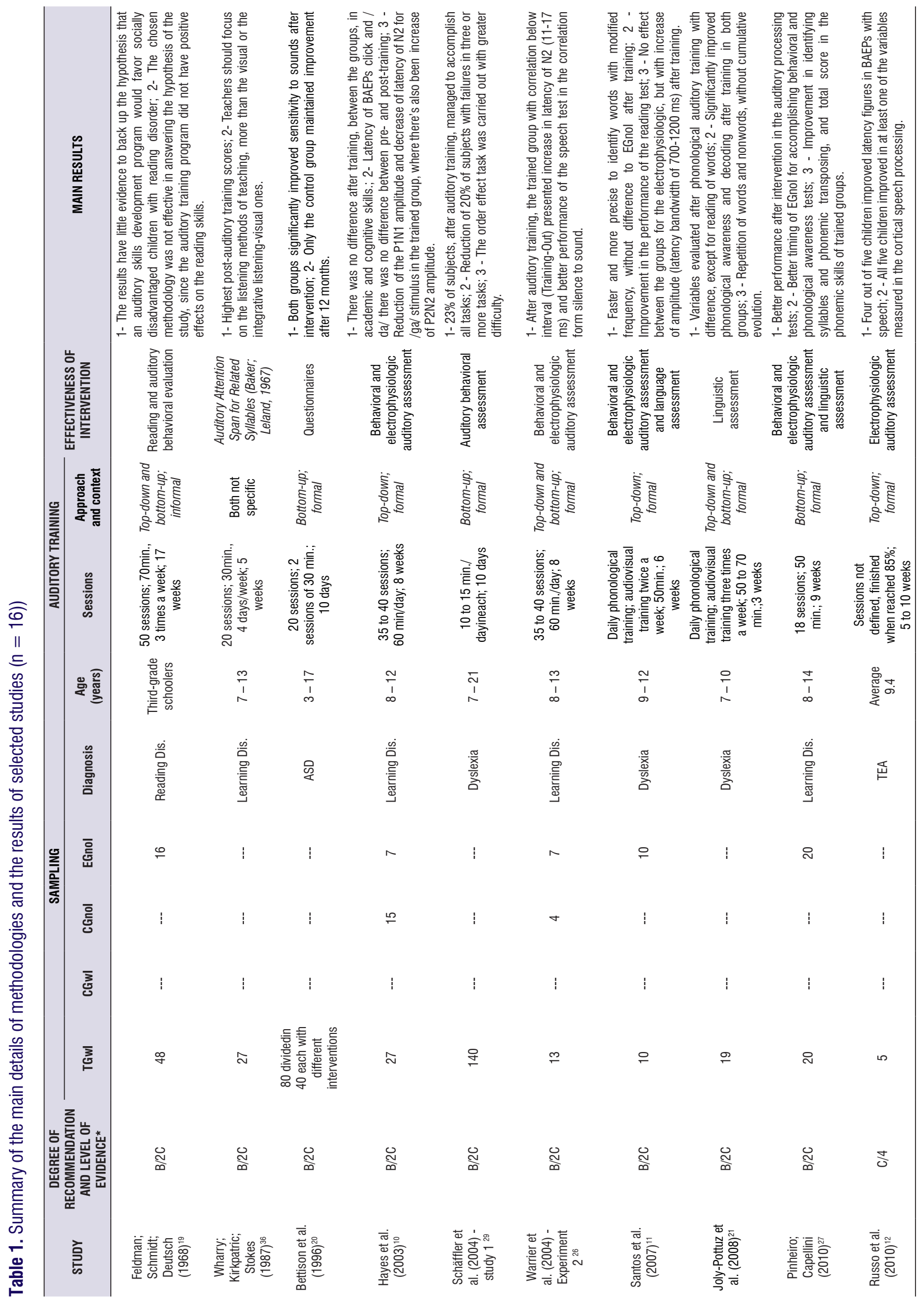




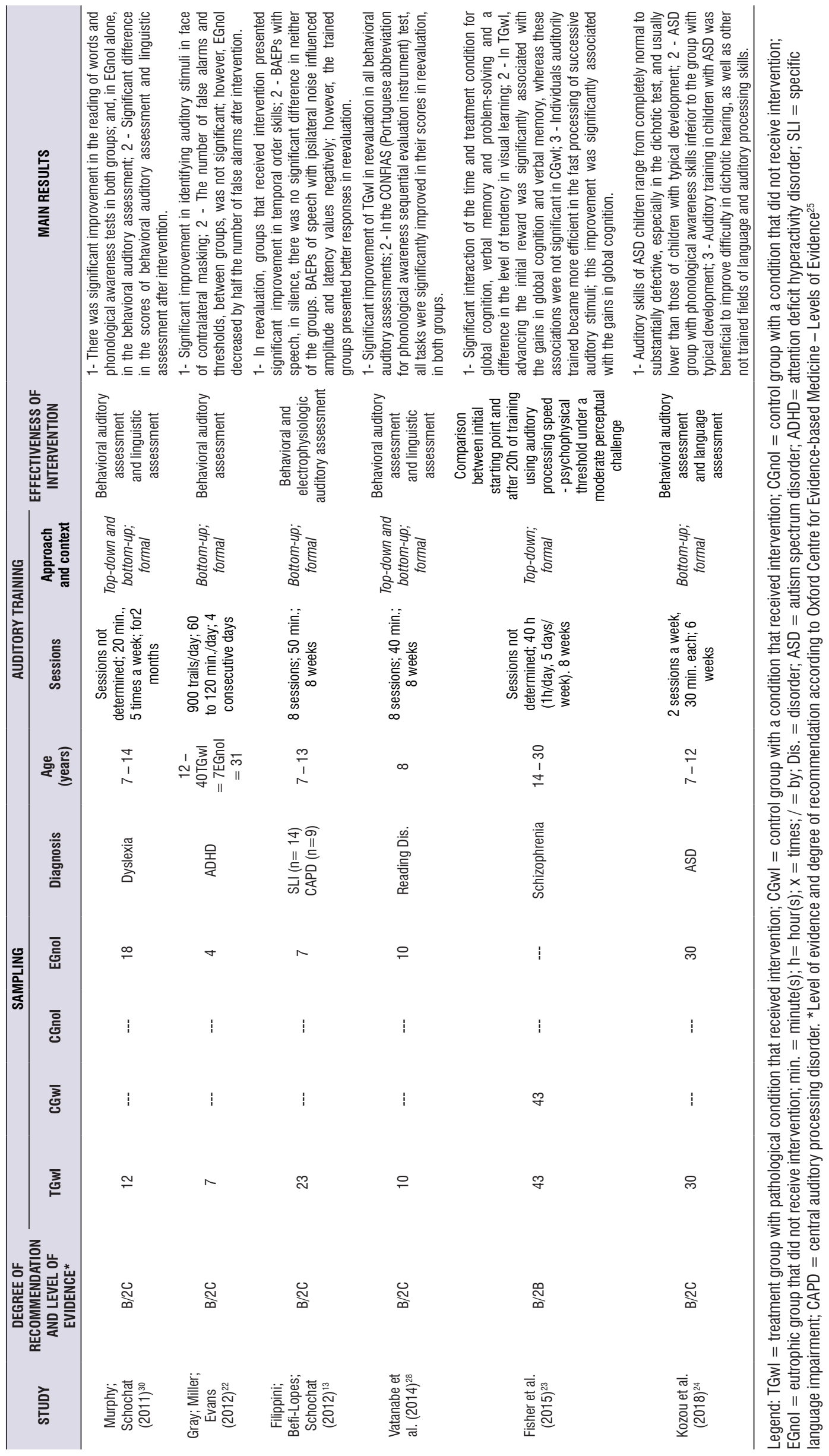


There has been noticed in literature paucity in studies that used AT as a means of intervention in children with psychiatric diagnoses or signs and symptoms. However, those that did refer to this methodology with such population report improvements in auditory, language, learning and cognitive skills.

The initial purpose of the searches carried out for the bibliographic survey of this integrative literature review was only the child psychiatric diagnosis associated with auditory training. However, it was noticed that a great majority of the references associated AT with signs and symptoms, which was then taken into account. The child psychiatric diagnosis poses a challenge, once great part of psychiatric disorders are diagnosed by the combination and intensity of signs, or objective findings characterized by the examining doctor, symptoms, through registered complaints, and functional loss $^{31,32}$. In spite of the assistance provided by classification systems, such as DSM and IDC, symptoms are overlapping and there are always individual and subjective aspects that act upon the patient's clinical results and diagnosis ${ }^{31}$.

In the studies included in this review, the variability of composition of the treatment groups shows that, for the studied population there's still no consensus in the researches to provide uniformity of these in relation to the control group. Aiming at being a parameter for controlling the evolution in the group with a specific psychiatric diagnosis or signs and symptoms, the control group is formed by subjects in the same condition, but divided in those that didn't receive intervention, those that were intervened with placebo, and those with typical development, either having or not received intervention. The literature does not contemplate a recommendation for studies with AT in regard to group composition; nevertheless, these characteristics refer not only to this population, but also to researches whose samples involve subjects with CAPD, the $\mathrm{Cl}$ and PSAP users, and/or aphasic subjects ${ }^{33-35}$.

The most prevalent methodological outline has a " $2 \mathrm{C}$ " level of evidence, followed by another two articles with "2B" and "C" levels of evidence, extremes in the classification of the selected ones. As for the degree of recommendation, grade "B" prevailed. Most important in this analysis is to identify that in $93.75 \%$ of the articles (15 out of 16), those with grade " $B$ " of recommendation 1, 0,11,13,19-24,26-30,36, presented believable results, i.e., their results may be generalized to the studied conditions, being thus useful for clinical decision-making.
The study with the lower degree of recommendation, classified as "C"12, suggests that there are minimal satisfactory evidences in the analysis of results, so that the benefits presented don't justify a generalization of the recommendation, once there's not enough sufficiently favorable evidence strength ${ }^{25}$. It's fitting to observe, since this classification system was chosen for its comprehensiveness to different studies, as it is in the specific case of the theme of therapeutics, that it does not differentiate studies with or without control groups, and, as a criteria for greater level of significance, it uses randomization in the sampling distribution, in which only one study presented this requisite for higher grading. Nonetheless, the choice for this system resulted from it being the most used in the different conditions of human health.

The most adopted auditory training approaches were the of the bottom-up type or the mixed, bottom-up and top-down, occurring respectively in $37.5 \%$ and $31.25 \%$ of the selected articles. This shows that the bottom-up intervention has been frequently used in this population, either associated or not with top-down mechanisms stimulation for a better approach and more comprehensive results, not only in the context of sensory stimulation, but also along with metalinguistic and metacognitive strategies.

Both approaches benefit from what underlies the AT theory, the neural plasticity, being the CANS capable of reorganizing at the cortical level through experiences and stimulation ${ }^{8}$. Plasticity in the context of the auditory system, which refers to alterations in nerve cells caused by environmental influences capable of showing behavioral changes, is distinguished in three different types, namely: 1) development plasticity; 2) compensated plasticity, resulting from an injury in the auditory system; 3) learning-related plasticity ${ }^{5,37}$.

This neural reorganization, in general, happens in two ways: it either activates neurons or connections previously inactive, or incites new neural connections to be formed ${ }^{5}$. According to the authors, these changes may be slow to happen, demanding more time and training, or they may be fast, without intervention, coming about only through development and/or maturation. Literature also reports that auditory cortical plasticity, as nerve cells improve by influence of the environment, brings about behavioral changes ${ }^{10,38}$.

The proposed AT carried out in the abovementioned approaches may further differ in relation to the context in which they were applied, formal or informal, which diverge as to the use of technological resources in order 
to acoustically control the stimuli presented during the training ${ }^{8,39}$. In the articles reviewed here, it was possible to identify that $87.5 \%$ made use of the application of AT in a formal context, which demonstrates greater zeal for the acoustic properties and characteristics of the stimuli used for the best effectiveness of the chosen AT approach.

Even though the intervention must be personalized for each case, the bottom-up and top-down training approaches are complementary and must be incorporated in order to maximize the effectiveness of the treatment ${ }^{7}$.

Observation of the patient's evolution is made possible by means of the registry and comparison of qualitative and quantitative data, both before and after auditory stimuli through $\mathrm{AT}^{33,40}$. Considered to be a key element for measuring the effects of any AT program, its effectiveness is thus indispensable ${ }^{5}$. In the selected studies, it was monitored by means of a pre- and postintervention analysis methodology, which was in great part monitored by the behavioral assessment of the auditory processing. There was also a variation in the use of questionnaires, and in the linguistic and electrophysiologic assessment of the auditory processing. To reveal the maturity of the auditory system and the level of neuroplasticity occurred during the auditory training is one of the main goals of the control of effectiveness in $A T^{5,41}$. Furthermore, the evolution in functional skills (e.g., auditory comprehension andoral linguistic processing in educational activities) is considered as a true measurement of the effectiveness of an AT program $^{39}$.

The articles analyzed in this integrative literature review, as well as most of the articles intending to study the effects of AT through a reevaluation after the training is carried out, measure it immediately. This is a gap that needs to be closed with new researches in studies that evaluate the effectiveness of training programs with $C P A D$, especially in the long term.

The amount of sessions, their frequency and duration were variables that wavered according to population, so that the AT approach would better suit the objective of the study. Even though it's accepted that, in order to increase neuroplasticity, intervention must be frequent, intense and challenging, it's justifiable that the doctor consider minimizing time and efforts necessary for promoting the same developmental scale of the patient, even when there's yet no recommendation with scientific evidence to determine the frequency and intensity of the AT intervention processes $^{39}$.

In summary, in the main results of the studies, regardless of the cases and the AT methodology used by the authors, the sampled subjects in the trained treatment groups presented improvement in linguistic and auditory skills, as compared to the evaluation carried out before the intervention, as well as in cognitive abilities. Only in the study authored by Feldmann; Schmidt; Deutsch ${ }^{19}$ the results presented little evidence to back up the author's initial hypothesis, whose auditory skills development program chosen for the study would work as a corrective measure for socially disadvantaged children with reading disorder.

Even if in the selected studies the results differed from each other, though congruous with the intervention model and intended objective for the studied population, the registry of the authors regarding observable evolutions is notorious, being them either significant or merely qualitatively described. The authors' conclusions refer to the goal of the applied AT, since the auditory stimulation, in order to restructure synaptic activity and provoke plasticity in the CANS ${ }^{36}$, resulted in improvements in the behavioral activities of the subjects. It's important to point out that these results were observed in a population with psychiatric diagnoses, in conditions that affect development. Thus, the auditory intervention process is a complementary strategy, not an exclusive one, in the cases in which there's medical recommendation for medicated treatment.

\section{CONCLUSION}

The bottom-up and the mixed (bottom-up and top-down) AT approaches were used in most of the studies, the formal context of training being the most frequent one. The protocols are heterogeneous in relation to the number and duration of sessions, as well as their frequency. Behavioral assessments were the most recurrent instruments for the control of effectiveness, followed by the combined use of behavioral and electrophysiologic assessments. The proposal of therapeutic intervention by means of AT in the population with diagnosis and signs and symptoms of child psychiatric has proved to be capable of promoting behavioral, auditory, cognitive and linguistic benefits in conditions such as dyslexia, ASD, schizophrenia, $A D H D$, learning disorder and language development disorder. 


\section{REFERENCES}

1. Katz J, Stecker N, Henderson D. Central auditory processing: a transdisciplinary view. St. Louis, MO: Mosby Year Book; 1992.

2. Musiek F, Chermak G. Handbook of (central) auditory processing disorder, volume 1: auditory neuroscience and diagnosis. Vol. 1. 2 ed. San Diego, CA: Plural Publishing Inc.; 2013.

3. Musiek FE, Baran JA, Shinn JB, Guenette L, Zaidan E, Weihing J. Central deafness: an audiological case study. Int J Audiol. 2007;46(8):433-41.

4. Kraus N, McGee T, Carrell TD, King C, Tremblay K, Nicol T. Central auditory system plasticity associated with speech discrimination training. $\mathrm{J}$ Cogn Neurosci. 1995;7(1):25-32.

5. Musiek F, Shinn J, Hare C. Plasticity, auditory training, and auditory processing disorders. Seminars in Hearing. 2002;23(4):263-76.

6. Bellis T. Assessment and management of central auditory processing disorders in the educational setting from science to practice. 2nd ed. San Diego, CA Plural Publishing Inc.; 2003.

7. American Academy of Audiology (AAA). Clinical practice guidelines: diagnosis, treatment and management of children and adults with central auditory processing disorder. In: 2010: https://audiology-web.s3.amazonaws.com/ migrated/CAPD\%20Guidelines\%208-2010. pdf5539952af956c79.73897613.pdf.

8. Chermak G, Musiek F. Auditory training: principles and approaches for remediating and managing auditory processing disorders. Seminars in Hearing. 2002;23(4):297-308.

9. Purdy SC, Kelly AS, Thorne PR. Auditory evoked potentials as measures of plasticity in humans. Audiol Neurootol. 2001;6(4):211-5.

10. Hayes EA, Warrier CM, Nicol TG, Zecker SG, Kraus $\mathrm{N}$. Neural plasticity following auditory training in children with learning problems. Clin Neurophysiol. 2003;114(4):673-84.

11. Santos A, Joly-Pottuz B, Moreno S, Habib $M$, Besson M. Behavioural and event-related potentials evidence for pitch discrimination deficits in dyslexic children: improvement after intensive phonic intervention. Neuropsychologia. 2007;45(5):1080-90.

12. Russo NM, Hornickel J, Nicol T, Zecker S, Kraus N. Biological changes in auditory function following training in children with autism spectrum disorders. Behav Brain Funct. 2010;6:60.
13. Filippini R, Befi-Lopes DM, Schochat E. Efficacy of auditory training using the auditory brainstem response to complex sounds: auditory processing disorder and specific language impairment. Folia Phoniatr Logop. 2012;64(5):217-26.

14. Polanczyk GV, Salum GA, Sugaya LS, Caye A, Rohde LA. Annual research review: A meta-analysis of the worldwide prevalence of mental disorders in children and adolescents. J Child Psychol Psychiatry. 2015;56(3):345-65.

15. Paula CS, Duarte CS, Bordin IAS. Prevalence of mental health problems in children and adolescents from the outskirts of Sao Paulo City: treatment needs and service capacity evaluation. Brazilian $\mathrm{J}$ Psychiatry. 2007;29(1):11-7.

16. Goodman R, Scott S. Childand adolescent psychiatry. 3 ed. London, UK: Wiley-Blackwell; 2012.

17. Atladottir HO, Gyllenberg D, Langridge A, Sandin $\mathrm{S}$, Hansen $\mathrm{SN}$, Leonard $\mathrm{H}$ et al. The increasing prevalence of reported diagnoses of childhood psychiatric disorders: a descriptive multinational comparison. Eur Child Adolesc Psychiatry. 2015;24(2):173-83.

18. Olfson M, Blanco C, Wang S, Laje G, Correll CU. National trends in the mental health care of children, adolescents, and adults by office-based physicians. JAMA Psychiatry. 2014;71(1):81-90.

19. Feldmann SC, Schmidt DE, Deutsch CP. Effect of auditory training on reading skills of retarded readers. Percept Mot Skills. 1968;26(2):467-80.

20. Bettison $\mathrm{S}$. The long-term effects of auditory training on children with autism. J Autism Dev Disord. 1996;26(3):361-74.

21. Joly-Pottuz B, Mercier M, Leynaud A, Habib M. Combined auditory and articulatory training improves phonological deficit in children with dyslexia. Neuropsychol Rehabil. 2008;18(4):402-29.

22. Gray L, Miller BS, Evans SW. Training children with $A D H D$ to minimize impulsivity in auditory contralateral masking. Int $\mathrm{J}$ Pediatr Otorhinolaryngol. 2012;76(4):483-7.

23. Fisher M, Loewy R, Carter C, Lee A, Ragland JD, Niendam $\mathrm{T}$ et al. Neuroplasticity-based auditory training via laptop computer improves cognition in young individuals with recent onset schizophrenia. Schizophr Bull. 2015;41(1):250-8.

24. Kozou H, Azouz HG, Abdou RM, Shaltout A. Evaluation and remediation of central auditory processing disorders in children with autism 
spectrum disorders. Int J Pediatr Otorhinolaryngol. 2018;104:36-42.

25. Howick J. Oxford Centre for Evidence-based Medicine - Levels of Evidence. 2009; http://www. cebm.net/index.aspx?o=1025.

26. Warrier CM, Johnson KL, Hayes EA, Nicol T, Kraus $\mathrm{N}$. Learning impaired children exhibit timing deficits and training-related improvements in auditory cortical responses to speech in noise. Exp Brain Res. 2004;157(4):431-41.

27. Pinheiro FH, Capellini SA. Treinamento auditivo em escolares com distúrbio de aprendizagem. Pró-Fono R. Atual. Cient. 2010;22(1):49-54.

28. Vatanabe TY, Navas ALGP, Mariano SPB, Murphy $\mathrm{CB}$, Durante AS. Performance of children with reading difficulties after auditory training. Audiol., Commun. Res. 2014;19(1):7-12.

29. Schaffler T, Sonntag J, Hartnegg K, Fischer B. The effect of practice on low-level auditory discrimination, phonological skills, and spelling in dyslexia. Dyslexia. 2004;10(2):119-30.

30. Murphy CF, Schochat E. Effect of nonlinguistic auditory training on phonological and reading skills. Folia Phoniatr Logop. 2011;63(3):147-53.

31. D’Abreu L. O desafio do diagnóstico psiquiátrico na criança. Contextos clínicos. 2012;5(1):2-9.

32. American Psychiatric Association (APA). Diagnostic and Statistical Manual of Mental Disorders. In. 5th ed. Washington, DC: American Psychiatric Association; 2013.

33. Wilson WJ, Arnott W, Henning C. A systematic review of electrophysiological outcomes following auditory training in school-age children with auditory processing deficits. Int $\mathrm{J}$ Audiol. 2013;52(11):721-30.

34. Barker F, MacKenzie E, Elliott L, de Lusignan S. Outcome measurement in adult auditory rehabilitation: a scoping review of measures used in randomized controlled trials. Ear Hear. 2015;36(5):567-73.

35. Woodhead ZV, Crinion J, Teki S, Penny W, Price CJ, Leff AP. Auditory training changes temporal lobe connectivity in 'Wernicke's aphasia': a randomised trial. J Neurol Neurosurg Psychiatry. 2017;88(7):586-94.

36. Wharry RE, Kirkpatrick SW, Stokes KD. Auditory training: effect on auditory retention with the learning disabled. Percept Mot Skills. 1987;65(3):1000.

37. Musiek F, Berge B. A neuroscience view of auditory training/stimulation and central auditory processing disorders. In: Masters MG, Stecker NA, Katz J (eds). Central auditory processing disorders mostly management. Boston: Allyn \& Bacon; 1998. p. 15-32.

38. Berlau KM, Weinberger NM. Learning strategy determines auditory cortical plasticity. Neurobiol Learn Mem. 2008;89(2):153-66.

39. Schochat $E$. Insights for management of processing disorders. Hear J. 2004;57(10):58.

40. Tawfik S, Mohamed Hassan D, Mesallamy R. Evaluation of long term outcome of auditory training programs in children with auditory processing disorders. Int $\mathrm{J}$ Pediatr Otorhinolaryngol. 2015;79(12):2404-10.

41. Chermak G. Neurobiological connections are key to APD. Hear J. 2004;57(4):58-9. 